\title{
Comparison of novel visceral obesity indexes with traditional obesity measurements in predicting of metabolically unhealthy nonobese phenotype in hemodialysis patients
}

\author{
${\text { Chaomin } \text { Zhou }^{1 *+} \mathbb{D} \text {, Yanzhe Peng }}^{1+}$, Wenyong Jiang ${ }^{2+}$, Jing Yuan ${ }^{1}$ and Yan Zha ${ }^{1 *}$
}

\begin{abstract}
Background: Normal-weight maintenance hemodialysis (MHD) patients with abdominal obesity exhibited a more proatherogenic profile than overweight and obesity patients with abdominal obesity, highlighting the importance of early identification of metabolically unhealthy nonobese (MUNO) in this population. Visceral fat accumulation plays a crucial role in the development of MUNO. Lipid accumulation product (LAP), visceral adiposity index (VAl) have been proved as reliable visceral obesity markers. The Chinese visceral adiposity index (CVAI) and a body shape index (ABSI) are newly discovered indexes of visceral obesity and have been reported to be associated with multiple metabolic disorders. There are limited studies investigating the associations between different visceral obesity indices and risk of MUNO, especially in hemodialysis patients. Moreover, no general agreement has been reached to date regarding which of these obesity indices performs best in identifying MUNO. We aimed to investigate the prevalence of MUNO in MHD patients and compare the associations between different adiposity indices (CVAI, ABSI,VAl, LAP, body mass index (BMI), waist circumference (WC) and waist-to-hip ratio (WHtR)) with MUNO risk in this population.
\end{abstract}

Methods: We conducted a multi-center cross-sectional study in Guizhou Province, Southwest China. 1302 nonobese adult MHD patients were included in our study. MUNO was defined as being nonobese and having the presence of $>=2$ components of metabolic syndrome (MetS). Nonobese was defined as BMl less than $25 \mathrm{~kg} / \mathrm{m}^{2}$. VAl, LAP, CVAl, ABSI, BMI, WC and WHtR were calculated. Logistic regression analyses and receiver operator curve (ROC) analyses were performed.

Results

65.6\% participants were metabolically unhealthy. The ROC curve analysis demonstrated that of the seven obesity indices tested, the VAI (AUC 0.84 for women and 0.79 for men) followed by LAP (AUC 0.78 for women and 0.72 for

\footnotetext{
*Correspondence: 723729549@qq.com; zhayan72@126.com

${ }^{\dagger}$ Chaomin Zhou, Yanzhe Peng and Wenyong Jiang contributed equally to this work.

'Renal Division, Department of Medicine, Guizhou Provincial People' s

Hospital, Guizhou Provincial Institute of Nephritic and Urinary Disease, No. 83,

Zhongshan Road, Guiyang, Guizhou, China

Full list of author information is available at the end of the article
}

(c) The Author(s). 2021 Open Access This article is licensed under a Creative Commons Attribution 4.0 International License, which permits use, sharing, adaptation, distribution and reproduction in any medium or format, as long as you give appropriate credit to the original author(s) and the source, provide a link to the Creative Commons licence, and indicate if changes were made. The images or other third party material in this article are included in the article's Creative Commons licence, unless indicated otherwise in a credit line to the material. If material is not included in the article's Creative Commons licence and your intended use is not permitted by statutory regulation or exceeds the permitted use, you will need to obtain permission directly from the copyright holder. To view a copy of this licence, visit http://creativecommons.org/licenses/by/4.0/ The Creative Commons Public Domain Dedication waiver (http://creativecommons.org/publicdomain/zero/1.0/) applies to the data made available in this article, unless otherwise stated in a credit line to the data. 
men) had the highest diagnostic accuracy for MUNO phenotype while ABSI exhibited the lowest AUC value for identifying MUNO phenotype

Conclusions: Metabolically unhealthy is highly prevalent in nonobese MHD patients. VAI and LAP outperformed CVAl in discriminating MUNO in MHD patients. Though ABSI could be a weak predictor of MUNO, it is not better than WHtR, WC and BMI.

Keywords: Visceral obesity indices, Metabolically unhealthy nonobese phenotype, Hemodialysis

\section{Background}

Obesity is highly prevalent in patients with chronic kidney disease (CKD), the prevalence of obesity in CKD appears to range from 35 to $44 \%$ [1]. Several studies have demonstrated that higher body mass index (BMI) is associated with decreased mortality in patients receiving maintenance hemodialysis (MHD) therapy, known as the obesity paradox [2]. However, the survival benefits associated with higher BMI may be attenuated in participants with metabolic abnormalities [3]. Actually, BMI is not an optimal indicator in terms of assessing cardiometabolic risk associated with increased adiposity as not all obese subjects assessed by BMI show increased risk for metabolic diseases. A subgroup of obese individuals is metabolically healthy, which has been referred as "metabolically healthy obese" (MHO) phenotype [4]. Similarly, not all lean subjects could avoid the development of metabolic abnormalities. A subgroup of relatively lean individuals is at increased risk of cardiometabolic disease and is metabolically unhealthy. This condition has been defined as "metabolically unhealthy nonobese" (MUNO) phenotype [5, 6]. As is well known, Asians are predisposed to visceral or abdominal obesity and at increased risks of metabolic diseases despite with low BMI. In this regard, it becomes indispensable to stratify subjects according to metabolic status rather than obesity. A recent study suggests that normal weight MHD patients with abdominal obesity exhibit a more proatherogenic profile and lower physical ability than overweight and abdominally obese patients [7]. We speculate that the results are mainly attributed to the metabolically unhealthy characteristics of the patients. In this regard, it becomes indispensable to identify MUNO individuals in this population.

Visceral fat accumulation plays a crucial role in the development of metabolic unhealthy phenotype [8]. Visceral adiposity index (VAI) and lipid accumulation product (LAP) have been verified as reliable visceral adiposity measures by lots of studies $[9,10]$. A body shape index (ABSI) and Chinese visceral adiposity index (CVAI) have been identified as two novel markers of visceral obesity recent years [11]. The new markers are reported to outperform other obesity markers in assessing all-cause mortality [12] and identifying type 2 diabetes as well as diabetic complications [13, 14]. Whether the new visceral obesity markers are associated with risk of MUNO phenotype remains unknown. Though many visceral obesity indices such as VAI, LAP and ABSI have been reported to be associated with multiple metabolic disorders, studies investigating the associations between these indices and MUNO phenotype are limited. Moreover, no general agreement has been reached to date regarding which of these indices performs best. In addition, whether the new markers perform better than the old ones as well as the traditional adiposity measures in the prediction of MUNO phenotype in MHD patients remain inconclusive.

This study aimed to investigate the prevalence of MUNO in MHD patients and further explore and compare the associations of novel visceral obesity markers and traditional obesity indices with risk of MUNO phenotype in this population.

\section{Methods}

\section{Study population}

A multicenter, cross-sectional study was conducted in Guizhou Province, Southwest China. Adult MHD patients who received 4-h standard twice to thrice-weekly dialysis treatment in 11 hemodialysis centers of Guizhou Province from June 1, 2017 to September 30, 2017 were recruited to our study. All these patients were clinically stable and underwent MHD for more than 3 months. A total of 2103 patients participated in our study voluntarily. We excluded participants who received HD treatment for less than 3 months. Subjects with obvious edema or ascites, hearing disabilities, physical deformities, language barriers and on any kind of nutritional support were also excluded from our study. Overweight or obese $\left(\mathrm{BMI}>\mathrm{or}=25 \mathrm{~kg} / \mathrm{m}^{2}\right)$ patients were also excluded from our final analysis. All participants provided written informed consent. This study was approved by the ethics committee of The People's Hospital of Guizhou province.

\section{Data collection}

As described in our previous research [15], information on age, sex, current or past cigarette smoking, educational attainment, history of current and previous illness, 
and medical treatment was acquired through a structured questionnaire, which is similar to that in the previous article. The questionnaires were completed by welltrained physicians through face-to-face interviews with the patients. We extracted the latest laboratory parameters from medical records. These laboratory parameters included fasting glucose, triglyceride (TG), total cholesterol (TC), high-density lipoprotein cholesterol (HDL-c), and low-density lipoprotein-cholesterol (LDL-c). Subjects who did not have a routinely blood test in the past 3 months were excluded in the final analysis.

\section{Measurements}

All measurements were performed after a routine HD session by well-trained nurses and physicians following standardized procedures. Height and weight were obtained while the participants were barefoot and in light clothing. BMI was calculated as weight $(\mathrm{kg})$ divided by the square of the height $\left(\mathrm{m}^{2}\right)$. WC was measured with an inelastic measuring tape in centimetres at the midpoint between the last rib and the iliac crest with the patients in standing positions. WHtR was calculated as the ratio between $\mathrm{WC}$ and height. Blood pressure (BP) was measured on the non-fistula arm in a standardized manner by physicians in the 11 hemodialysis centers with an automatic manometer and the average of 3 readings was used in the final analysis. Pre-dialysis BP was measured before hemodialysis in a sitting position after a rest of $\geqslant$ 5 min.

\section{Calculation of anthropometric indices}

LAP [10], CVAI [16], VAI [10] and ABSI were calculated according to the following formulas:

$$
\begin{aligned}
& \mathrm{LAP}_{\text {men }}=(\mathrm{WC}(\mathrm{cm})-65) \times \mathrm{TG}(\mathrm{mmol} / \mathrm{l}) ; \\
& \mathrm{LAP}_{\text {women }}=(\mathrm{WC}(\mathrm{cm})-58) \times \mathrm{TG}(\mathrm{mmol} / \mathrm{l}) ; \\
& \mathrm{CVAI}_{\text {men }}=-267.93+0.68 \times \mathrm{age}+0.03 \times \mathrm{BMI}\left(\mathrm{kg} / \mathrm{m}^{2}\right)+ \\
& 4.00 \times \mathrm{WC}(\mathrm{cm})+22.00 . \\
& \times \log 10 \mathrm{TG}(\mathrm{mmol} / \mathrm{L})-16.32 \times \mathrm{HDL}-\mathrm{C}(\mathrm{mmol} / \mathrm{L}) ; \\
& \mathrm{CVAI}_{\text {women }}=-187.32+1.71 \times \mathrm{age}+4.23 \times \mathrm{BMI}\left(\mathrm{kg} / \mathrm{m}^{2}\right)+ \\
& 1.12 \times \mathrm{WC}(\mathrm{cm})+39.76 . \\
& \times \log 10 \mathrm{TG}(\mathrm{mmol} / \mathrm{L})-11.66 \times \mathrm{HDL}-\mathrm{C}(\mathrm{mmol} / \mathrm{L}) ; \\
& \mathrm{VAI}_{\text {men }}=\mathrm{WC}[39.68+(1.88 \times \mathrm{BMI})] \times \mathrm{TG} 1.03 \times \\
& 1.31 \mathrm{HDL} ; \\
& \mathrm{VAI}_{\text {women }}=\mathrm{WC}[36.58+(1.89 \times \mathrm{BMI})] \times \mathrm{TG} 0.81 \times \\
& 1.52 \mathrm{HDL} ; \\
& \mathrm{ABSI}^{2} \mathrm{WC}(\mathrm{m}) /\left[\mathrm{BMI}^{2 / 3}\left(\mathrm{~kg} / \mathrm{m}^{2}\right) \times \mathrm{height}^{1 / 2}(\mathrm{~m})\right] ;
\end{aligned}
$$

\section{Definition of metabolically unhealthy nonobese phenotype}

Subjects are considered to have MUNO phenotype if they have a BMI that is less than $25 \mathrm{~kg} / \mathrm{m}^{2}$ [9]. Furthermore, they must meet with two or more of the following abnormalities at the same time: (1) systolic BP $\geqslant 130 \mathrm{mmHg}$ and/or a diastolic $\mathrm{BP} \geqslant 85 \mathrm{mmHg}$, or use of antihypertensive drugs; (2) TG $\geqslant 1.7 \mathrm{mmol} / \mathrm{l} ;$ (3) HDL$\mathrm{C}<1.0 \mathrm{mmol} / \mathrm{l}$ in men and $<1.3 \mathrm{mmol} / \mathrm{l}$ in women or taking lipid-lowering drugs for the indication of hyperlipidemia; (4) fasting plasma glucose (FPG) $\geqslant 5.6 \mathrm{mmol} / \mathrm{l}$ or on antidiabetic treatment for raised blood glucose [17].

\section{Statistical analysis}

Continuous variables were presented as medians (interquartile range) due to their skewed distribution. MannWhitney $U$ test was used to compare differences between metabolically healthy nonobese (MHNO) and MUNO patients. Categorical variables were expressed as frequency and proportion and differences between the two groups were analyzed by Chi-square tests. Quartiles of CVAI, VAI, LAP, ABSI, BMI, WC and WHtR were calculated and the lowest quartiles of the indexes were set as the reference. Logistic regression analyses were applied to evaluate the independent association of different adiposity indices with MUNO phenotype. The receiver operating characteristic (ROC) curves were built and areas under ROC curves (AUROCs) were calculated to estimate the diagnostic accuracy of the seven obesity indexes for MUNO phenotype. The optimal cut-off points for different adiposity indices were calculated according to the Youden indexes. Significance tests for comparison of AUCs from different obesity indices were assessed by the method described by DeLong [18]. Statistical analyses were performed with the statistical software SPSS version 16.0. Significance tests for comparison of AUCs were performed using MedCalc version 13.0 for Windows (MedCalc Software, Mariakerke, Belgium).

\section{Results}

1302 nonobese individuals ( $54 \pm 16$ years), ranging in age from 18 to 90 years, met the inclusion criteria and were included in our final analysis. Among these individuals, 854(65.6\%) participants met the criteria of MUNO phenotype and 448 subjects were metabolically healthy. The basic characteristics of the study population were listed in Table 1. Compared with metabolically healthy nonobese (MHNO) individuals, patients with the MUNO phenotype seemed to be younger and had significantly higher WC, BMI, WHtR, CVAI, VAI, LAP, FPG and TG, but lower HDL-C and diastolic BP. In addition, subjects with the MUNO phenotype exhibited a higher prevalence of history of hypertension and diabetes. No significant differences were found between the two groups for levels of LDL-C, TC, systolic BP and educational status.

\section{Risk of MUNO phenotype by quartiles of different adiposity indices}

As shown in Table 2, all adiposity indices included in our study were independently associated with MUNO 
Table 1 Baseline characteristics of the study population

\begin{tabular}{|c|c|c|c|}
\hline Characteristics & MHNO $(n=448)$ & MUNO $(n=854)$ & $P$ value \\
\hline$\overline{\text { Age }(y r)}$ & $59(47,70)$ & $52(39,65)$ & 0.005 \\
\hline Male n(\%) & $296(66.1 \%)$ & $489(57.3 \%)$ & 0.001 \\
\hline History of hypertension (\%) & $244(63.7 \%)$ & $273(88.9 \%)$ & $<0.001$ \\
\hline History of diabetes (\%) & $446(13.0 \%)$ & $596(35.8 \%)$ & $<0.001$ \\
\hline History of smoking (\%) & $214(41.5 \%)$ & $146(35.6 \%)$ & 0.04 \\
\hline Educational status ( $\geq$ junior high school)(\%) & $34.8 \%$ & $36.3 \%$ & 0.35 \\
\hline Systolic BP (mmHg) & $140(125,154)$ & $140(127,154)$ & 0.9 \\
\hline Diastolic BP (mmHg) & $80.0(70,89.5)$ & $77(68,88)$ & 0.003 \\
\hline WC $(\mathrm{cm})$ & $77.5(72,84.2)$ & $82(75.5,87)$ & $<0.001$ \\
\hline BMI $\left(\mathrm{kg} / \mathrm{m}^{2}\right)$ & $20.35(18.91,22.20)$ & $21.46(19.63,23.06)$ & $<0.001$ \\
\hline WHtR & $0.48(0.45,0.53)$ & $0.51(0.47,0.54)$ & $<0.001$ \\
\hline CVAl & $59.97(31.24,91.72)$ & $87.47(57.04,112.64)$ & $<0.001$ \\
\hline VAl & $1.17(0.82,1.59)$ & $2.40(1.43,3.73)$ & $<0.001$ \\
\hline LAP & $15.19(8.58,23.94)$ & $29.50(16.37,51.94)$ & $<0.001$ \\
\hline ABSI & $0.082(0.078,0.087)$ & $0.084(0.080,0.088)$ & $<0.001$ \\
\hline FPG (mmol/L) & $4.73(4.35,5.17)$ & $6.22(4.99,8.10)$ & $<0.001$ \\
\hline TG $(\mathrm{mmol} / \mathrm{L})$ & $1.05(0.81,1.33)$ & $1.62(1.1,2.31)$ & $<0.001$ \\
\hline $\mathrm{HDL}-\mathrm{C}(\mathrm{mmol} / \mathrm{L})$ & $1.31(1.12,1.55)$ & $1.02(0.84,1.26)$ & $<0.001$ \\
\hline LDL-C (mmol/L) & $2.10(1.64,2.75)$ & $2.20(1.70,2.78)$ & 0.24 \\
\hline TC (mmol/L) & $3.87(3.24,4.42)$ & $3.89(3.31,4.60)$ & 0.18 \\
\hline
\end{tabular}

Abbreviations: WC waist circumference, BMI body mass index, WHtR waist-height ratio, CVAI Chinese visceral adiposity index, VAl visceral adiposity index, LAP lipid accumulation product, $A B S I$ a body shape index, $B P$ Blood pressure, $F B G$ fasting blood-glucose, $T G$ triglyceride, $H D L-c$ high-density lipoprotein cholesterol, $L D L-c$ low-density lipoprotein-cholesterol, TC total cholesterol

phenotype risk, even after adjustment for potential intermediate variables such as age, sex, educational status and history of smoking. The adjusted ORs for MUNO phenotype rose progressively with increasing quartiles of adiposity indices except for ABSI.

\section{ROC analyses for different adiposity indices to predict the risk of MUNO phenotype}

As shown in Table 3 and Fig. 1, all the obesity indices included in the study were able to discriminate
MUNO phenotype (all area under the ROC curves [AUCs] $>0.5, P<0.05)$. The ROC curve analysis demonstrated that of the seven obesity indices tested, the VAI (AUC 0.84 for women and 0.79 for men) followed by LAP (AUC 0.78 for women and 0.72 for men) had the highest diagnostic accuracy for MUNO phenotype while ABSI was the least accurate predictor of MUNO phenotype for both men and women. The best VAI value for diagnosis of MUNO phenotype was 1.71 (sensitivity 0.60 , specificity 0.86 )

Table 2 Odds ratio $(95 \% \mathrm{Cl})$ of the presence of MUNO for different adiposity indices after adjustment of age, sex, educational status and history of smoking

\begin{tabular}{lllll}
\hline Obesity indexes & Quartile 1 & Quartile 2 & Quartile 3 & Quartile 4 \\
\hline BMI & 1 & $1.01(0.70-1.45)$ & $2.07(1.41-3.05)^{*}$ & $2.20(1.48-3.28)^{*}$ \\
WC & 1 & $1.53(1.05-2.22)^{*}$ & $2.73(1.82-4.07)^{*}$ & $2.96(1.94-4.51)^{*}$ \\
WHtR & 1 & $1.74(1.19-2.54)^{*}$ & $2.49(1.69-3.66)^{*}$ & $3.11(2.00-4.85)^{*}$ \\
VAI & 1 & $2.35(1.60-3.44)^{*}$ & $10.01(6.38-15.71)^{*}$ & $88.18(34.56-225.02)^{*}$ \\
CVAl & 1 & $3.39(2.19-5.25)^{*}$ & $8.08(4.82-13.55)^{*}$ & $13.23(7.39-23.68)^{*}$ \\
ABSI & 1 & $1.75(1.21-2.55)^{*}$ & $2.08(1.42-3.06)^{*}$ & $1.97(1.33-2.92)^{*}$ \\
LAP & 1 & $1.76(1.82-4.69)^{*}$ & $3.51(2.35-5.22)^{*}$ & $30.16(15.81-57.55)^{*}$ \\
\hline
\end{tabular}

The between cut points are 19.27, 21.17, 22.87 for BMl; 74.50, 80.35, 86.23for WC; $0.46,0.50,0.54$ for WHtR; 1.14, 1.74, 3.09 for VAl; 48.4, 79.33, 107.4 for CVAl; $0.079,0.083,0.088$ for ABSI; $12.80,23.19,39.68$ for LAP; ${ }^{*} P<0.05, ※<0.001$

Abbreviations: $B M I$ body mass index, WC waist circumference, WHtR waist-to-height ratio, VAl visceral adiposity index, CVAI Chinese visceral adiposity index, $A B S I$ a body shape index, LAP lipid accumulation product 
Table 3 Comparison of diagnostic performance of novel visceral obesity indices with old ones and traditional adiposity indices

\begin{tabular}{|c|c|c|c|c|c|c|}
\hline Adiposity indices & AUC $(95 \% \mathrm{Cl})$ & $p$ & Cut-off & Sensitivity & Specificity & Youden Index \\
\hline \multicolumn{7}{|l|}{ Males } \\
\hline $\mathrm{BMI}$ & $0.58(0.55-0.62)$ & $<0.001$ & 20.69 & 0.65 & 0.51 & 0.16 \\
\hline WC & $0.60(0.57-0.64)$ & $<0.001$ & 81.5 & 0.59 & 0.62 & 0.21 \\
\hline $\mathrm{WHtR}$ & $0.61(0.57-0.64)$ & $<0.001$ & 0.48 & 0.68 & 0.53 & 0.20 \\
\hline VAl & $0.79(0.76-0.82)$ & $<0.001$ & 1.71 & 0.60 & 0.86 & 0.46 \\
\hline CVAl & $0.65(0.62-0.69)$ & $<0.001$ & 72.39 & 0.67 & 0.58 & 0.25 \\
\hline$A B S I$ & $0.56(0.53-0.60)$ & 0.005 & 0.080 & 0.73 & 0.41 & 0.14 \\
\hline LAP & $0.72(0.68-0.75)$ & $<0.001$ & 24.38 & 0.53 & 0.80 & 0.34 \\
\hline \multicolumn{7}{|l|}{ Females } \\
\hline $\mathrm{BMI}$ & $0.64(0.60-0.69)$ & $<0.001$ & 20.96 & 0.56 & 0.72 & 0.27 \\
\hline WC & $0.64(0.60-0.68)$ & $<0.001$ & 72.8 & 0.82 & 0.41 & 0.23 \\
\hline $\mathrm{WH} \mathrm{tR}$ & $0.62(0.58-0.66)$ & $<0.001$ & 0.47 & 0.83 & 0.38 & 0.21 \\
\hline $\mathrm{VAl}$ & $0.84(0.80-0.87)$ & $<0.001$ & 2.00 & 0.72 & 0.87 & 0.59 \\
\hline CVAl & $0.70(0.66-0.74)$ & $<0.001$ & 72.22 & 0.61 & 0.71 & 0.33 \\
\hline ABSI & $0.56(0.52-0.61)$ & 0.03 & 0.084 & 0.53 & 0.61 & 0.14 \\
\hline LAP & $0.78(0.73-0.81)$ & $<0.001$ & 30.08 & 0.60 & 0.84 & 0.43 \\
\hline
\end{tabular}

Abbreviations: $B M I$ body mass index, WC waist circumference, $W H t R$ waist-to-height ratio, VAl visceral adiposity index; CVAI Chinese visceral adiposity index, $A B S I$ a body Abbreviations shape index, LAP lipid accumulation product

for men and 2.0 (sensitivity 0.72 , specificity 0.87 ) for women.

\section{Significance tests of AUCs from different obesity indices for prediction of MUNO phenotype}

Differences between the AUCs of VAI with LAP; WHtR with CVAI and ABSI; WC with WHtR, ABSI and CVAI; BMI with WC, WHtR and ABSI were further tested as their 95\% CI were overlapped. BMI showed similar discriminative power with WC, WHtR and ABSI in both genders based on non-significant differences in AUC tests (shown in Table 4). WC performed better than WHtR in predicting MUNO phenotype in women $(P=$ $0.02)$, while WC showed similar discriminative power with WHtR in men $(P=0.87)$. CVAI showed higher AUC than WC and WHtR for detection of MUNO in both men and women. VAI outperformed LAP showed lower AUC (in comparison with VAI), whereas WC and WHtR showed better predictive capacity of MUNO than $\operatorname{ABSI}(P<0.05)$.

\section{Discussion}

Our study demonstrated that $65.6 \%$ participants were metabolically unhealthy, despite with relatively low BMI. All the seven adiposity indices included in our study were independently associated with increased risk for MUNO phenotype. VAI and LAP showed significantly higher AUCs than CVAI while ABSI was inferior to traditional anthropometric indices in discriminating MUNO phenotype.
A more proatherogenic profile in MHD patients with normal weight abdominal obesity than obese or overweight MHD patients highlighted the importance of exploring the relationship between visceral/abdominal obesity and metabolically unhealthy in nonobese MHD patients [7]. Previous studies have suggested that visceral obesity contributes a lot to the development of MUNO phenotype [19]. Searching for simple and effective surrogate markers of visceral obesity has acquired more and more attentions since direct estimation of visceral fat demands imaging diagnostic tests which are costly and have low availability for epidemiological use. VAI and LAP are two reliable visceral obesity indices developed and validated in Caucasian population. But differences in body fat distribution should be considered among different ethnicity. It is widely known Chinese are more prone to be visceral obese despite having relatively low BMI. CVAI, a new visceral obesity index developed and validated in Chinese population, was found to be superior to other obesity markers in identifying cardiometabolic risk factors [16, 20-22]. However, CVAI is inferior to old visceral obesity indices such as VAI and LAP in discriminating MUNO phenotype in our study. In fact, the superiority of VAI in assessing metabolic risk has been reported by lots of studies $[17,23]$. Our finding that VAI followed by LAP, CVAI presented the highest AUROC for MUNO phenotype highlighted the supremacy of VAI in predicting MUNO phenotype. Similar to the general population, increasing BMI demonstrated a positive association with the MUNO phenotype in our study. This 

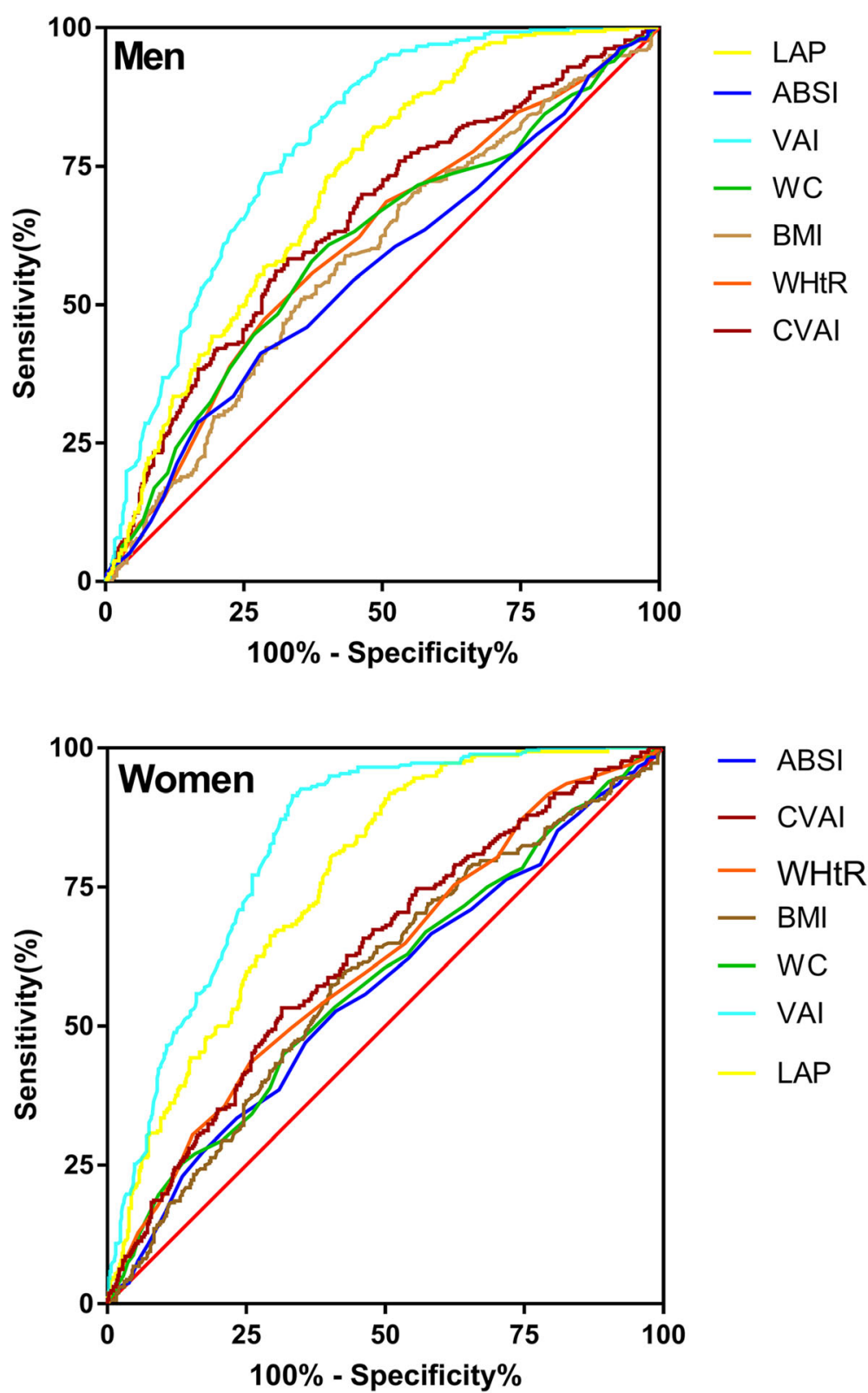

Fig. 1 ROC curves of the seven obesity indices to diagnose MUNO phenotype in men and women. Legend: BMI, body mass index; WC, waist circumference; WHtR, waist-to-height ratio;VAl, visceral adiposity index; CVAl, Chinese visceral adiposity index; ABSI, a body shape index; LAP, lipid accumulation product. ROC, receiver operating characteristic

was somewhat consistent with a previous study which demonstrated that normal-weight patients on hemodialysis with abdominal obesity exhibited a more proatherogenic profile than abdominal obesity patients with overweight and obesity [7]. Participants with higher BMI might be abdominal obese as most of the participants were middle-aged (mean age, $54 \pm 16$ years), and as we all known, middle-aged people were prone to abdominal obesity. Consistent with our study, a previous study also suggested that VAI followed by LAP was a better indicator of MUNO phenotype compared to BMI, WC and WHtR [10].

Exact mechanisms accounting for the different predictive value of CVAI in our study and previous studies are not known. Fat distribution may vary in different diseases and the characteristics of the study population are different. Participants with MUNO phenotype were relatively younger than those with $\mathrm{MHNO}$ phenotype in our study. A previous study has suggested that visceral adipose tissue (VAT) was not associated with incident 
Table 4 Significance tests for comparison of AUCs from different obesity indices

\begin{tabular}{llllll}
\hline Characteristics & \multicolumn{1}{l}{ Male } & & \multicolumn{2}{l}{ Female } \\
\cline { 2 - 3 } & $\boldsymbol{Z}$ & $\boldsymbol{P}$ & & $\boldsymbol{Z}$ \\
\hline BMI vs WC & 0.993 & 0.32 & & 0.457 & 0.65 \\
BMI vs WHtR & 1.111 & 0.27 & & 0.377 & 0.71 \\
BMI vs ABSI & 1.012 & 0.31 & 1.307 & 0.19 \\
WC vs WHtR & 0.158 & 0.87 & 2.276 & 0.02 \\
WC vs ABSI & 2.776 & 0.006 & 3.260 & 0.0001 \\
WC vs CVAl & 6.223 & $<0.0001$ & 2.116 & $<0.0001$ \\
WHtR vs CVAl & 4.481 & $<0.0001$ & 3.054 & 0.03 \\
WHtR vs ABSI & 2.776 & 0.006 & & 2.013 & 0.035 \\
LAP vs VAl & 4.487 & $<0.0001$ & 3.306 & $<0.0001$ \\
\hline
\end{tabular}

Abbreviations: $B M I$ body mass index, WC waist circumference, WHtR waist-toheight ratio, VAl visceral adiposity index; CVAl Chinese visceral adiposity index, $A B S I$ a body Abbreviations shape index, $L A P$ lipid accumulation product

atherosclerotic cardiovascular disease events in older men [24]. We speculated that VAT might play a protective role in metabolic risk in relatively older MHD patients. CVAI is calculated based on age, BMI, WC, TG, and HDL-C, but the establishment of VAI is not affected by age. Therefore, VAI outperformed CVAI as it is not affected by age in identifying metabolically unhealthy obesity in our study. Furthermore, though CVAI has been proposed to be a reliable Chinese-based marker of visceral obesity, only a small group of the subjects in the study of Xia et al. received abdominal computed tomography $(\mathrm{CT})$, which served as a gold method for visceral fat assessment [16]. Furthermore, AUROCs of WC and BMI for visceral fat evaluation in their study were even higher than that of VAI, which implied WC and BMI were better predictors of visceral obesity than VAI. This is obviously inconsistent with previous researches. More studies that directly measure visceral fat areas in Chinese population are needed to validate the predictive value of CVAI in evaluating visceral obesity.

ABSI, another new marker of visceral obesity, was reported to be a stronger predictor of diabetes than BMI [25] and was superior to traditional measures of adiposity in assessing carotid atherosclerosis [26]. In addition, ABSI achieves better mortality risk stratification than abdominal obesity indices [12]. However, the AUROC of ABSI for diagnosis of MUNO phenotype in our study was the lowest. Comparison of AUCs showed that WC and WHtR performed better than ABSI in discriminating MUNO while BMI share similar AUC with ABSI. In accordance with our results, a previous study suggested that VAI was superior to ABSI, WC, BMI and other anthropometric indices in assessing the risk of metabolic syndrome (MetS) [27]. As we all known, patients with MetS share similar metabolic profiles to those with MUNO phenotype. Our results were further strengthened by a previous study demonstrating that ABSI was a weaker predictor of MetS than WC and BMI [28]. Exact mechanisms accounting for the disadvantage of ABSI in predicting MUNO phenotype in our study are unknown. It might be attribute to the fact that ABSI doesn't take TG into account, but increased TG is associated with insulin resistance even in subjects with normal BMI and normal glucose tolerance [29] and insulin resistance is the core characteristic of MUNO phenotype. Furthermore, many studies have suggested that indexes composed of TG are very suitable for the identification of metabolically unhealthy individuals [30].

Several limitations of our study should be considered when interpreting the findings. Firstly, the crosssectional nature of the study disabled us to make causality between the obesity indices and MUNO risk. Secondly, high blood pressure is a key factor associates with the definition of MUNO phenotype. Most dialysis patients in our study had hypertension and this might attribute to the kidney diseases but not metabolic abnormality. Thus, the prevalence of MUNO might be exaggerated. Further studies are needed to clarify whether the definition of MUNO phenotype is suitable for dialysis patients. Thirdly, since mean age of our patients were $54 \pm 16$ years, our results and conclusions might be more suitable for middle-aged and elderly Chinese MHD patients. Fifth, some of the components included in the investigated indices are identical to the components included in the MUNO criteria. The mathematical coupling can inflate regression coefficients and AUCs, so the results should be interpreted with caution. Extrapolating our findings to other populations should be interpreted cautiously.

\section{Conclusions}

Metabolically unhealthy is highly prevalent in nonobese MHD patients. VAI and LAP outperformed CVAI in discriminating MUNO phenotype in MHD patients. Though ABSI could be a weak predictor of MUNO phenotype, it is not better than WHtR, WC and BMI.

\section{Abbreviations \\ BMI: Body mass index; WC: Waist circumference; WHtR: Waist-to-height ratio; VA: Visceral adiposity index; CVAl: Chinese visceral adiposity index; ABSI: A body abbreviations shape index; LAP: Lipid accumulation product; MHD: Maintenance hemodialysis; MUNO: Metabolically unhealthy nonobese; MetS: Metabolic syndrome; TG: Triglyceride; TC: Total cholesterol; HDL- c: High-density lipoprotein cholesterol; LDL-c: Low-density lipoprotein- cholesterol; ROC: Receiver operating characteristic}

Acknowledgements

We are grateful to participants in this study.

\section{Authors' contributions}

ZY oversaw the entire project and revised the manuscript. ZCM contributed to the conception and design of the study and analyzed the data and drafted the article. JWY and PYZ contributed to the conception and design of the study, they also contributed to the acquisition of data used in the 
study and revised the article. YJ contributed to the acquisition of data used in the study. All authors have read and approved the manuscript.

\section{Funding}

The present study was supported by the following Science Foundation: 1. High-level innovative personnel program of GuiZhou Province No.

\section{【2018】5636;}

2. Hospital Funds for Young Scientists of Guizhou Provincial People' s Hospital (GZSYQN [2016]11).

\section{Availability of data and materials}

The datasets used and/or analysed during the current study are available from the corresponding author on reasonable request.

\section{Declarations}

\section{Ethics approval and consent to participate}

Ethics Committee of The People' s Hospital of Guizhou province approved the study. This study was performed fulfilling the principles of Helsinki Declaration. All participants provided written informed consent prior to participation in this study.

\section{Consent for publication}

Not Applicable.

\section{Competing interests}

The authors have no conflict of interest to declare.

\section{Author details}

${ }^{1}$ Renal Division, Department of Medicine, Guizhou Provincial People' s Hospital, Guizhou Provincial Institute of Nephritic and Urinary Disease, No. 83, Zhongshan Road, Guiyang, Guizhou, China. 'Renal Division, The First People's Hospital of Guiyang, Guiyang, Guizhou, China.

\section{Received: 15 March 2021 Accepted: 28 November 2021}

\section{Published online: 19 December 2021}

\section{References}

1. Martin WP, Bauer J, Coleman J, Dellatorre-Teixeira L, Reeve JLV, Twomey PJ, et al. Obesity is common in chronic kidney disease and associates with greater antihypertensive usage and proteinuria: evidence from a crosssectional study in a tertiary nephrology Centre. Clin Obes. 2020;10(6): e12402. https://doi.org/10.1111/cob.12402.

2. Kim CS, Han K-D, Choi HS, Bae EH, Ma SK, Kim SW. Association of Body Mass Index and Waist Circumference with all-cause mortality in hemodialysis patients. J Clin Med. 2020;9(5):1289. https://doi.org/10.3390/jcm9051289.

3. Hanks LJ, Tanner RM, Muntner P, Kramer H, McClellan WM, Warnock DG, et al. Metabolic subtypes and risk of mortality in normal weight, overweight, and obese individuals with CKD. Clin J Am Soc Nephrol. 2013;8(12):2064-71. https://doi.org/10.2215/CJN.00140113.

4. Phillips CM. Metabolically healthy obesity: definitions, determinants and clinical implications. Rev Endocr Metab Disord. 2013;14(3):219-27. https:// doi.org/10.1007/s11154-013-9252-x.

5. Mathew H, Farr OM, Mantzoros CS. Metabolic health and weight: understanding metabolically unhealthy normal weight or metabolically healthy obese patients. Metabolism. 2016;65(1):73-80. https://doi.org/10.101 6/j.metabol.2015.10.019.

6. Ding C, Chan Z, Magkos F. Lean, but not healthy: the 'metabolically obese, normal-weight' phenotype. Curr Opin Clin Nutr Metab Care. 2016:19(6):40817. https://doi.org/10.1097/MC0.0000000000000317.

7. Beberashvili I, Azar A, Abu HR, Sinuani I, Feldman L, Maliar A, et al. Abdominal obesity in normal weight versus overweight and obese hemodialysis patients: associations with nutrition, inflammation, muscle strength, and quality of life. Nutrition. 2019;59:7-13. https://doi.org/10.1016/ j.nut.2018.08.002

8. Hwang YC, Hayashi T, Fujimoto WY, Kahn SE, Leonetti DL, McNeely MJ, et al. Visceral abdominal fat accumulation predicts the conversion of metabolically healthy obese subjects to an unhealthy phenotype. Int Obes. 2015;39(9):1365-70. https://doi.org/10.1038/ijo.2015.75.

9. Amato MC, Giordano C, Galia M, Criscimanna A, Vitabile S, Midiri M, et al. Visceral adiposity index: a reliable indicator of visceral fat function associated with cardiometabolic risk. Diabetes Care. 2010;33(4):920-2. https://doi.org/10.2337/dc09-1825.

10. Du T, Yu X, Zhang J, Sun X. Lipid accumulation product and visceral adiposity index are effective markers for identifying the metabolically obese normal-weight phenotype. Acta Diabetol. 2015;52(5):855-63. https://doi. org/10.1007/s00592-015-0715-2.

11. Han M, Qin P, Li Q, Qie R, Liu L, Zhao Y, et al. Chinese visceral adiposity index: a reliable indicator of visceral fat function associated with risk of type 2 diabetes. Diabetes Metab Res Rev. 2021;37(2):e3370. https://doi.org/10.1 002/dmrr.3370.

12. Christakoudi S, Tsilidis KK, Muller DC, Freisling H, Weiderpass E, Overvad K, et al. A body shape index (ABSI) achieves better mortality risk stratification than alternative indices of abdominal obesity: results from a large European cohort. Sci Rep. 2020;10(1):1-15. https://doi.org/10.1038/s41598-020-71302-5.

13. Han M, Qin P, Li Q, Qie R, Liu L, Zhao Y, et al. Chinese visceral adiposity index: A reliable indicator of visceral fat function associated with risk of type 2 diabetes. Diabetes/metabolism Res Rev. 2020;37(2):e3370.

14. Wan H, Wang Y, Xiang Q, Fang S, Chen Y, Chen C, et al. Associations between abdominal obesity indices and diabetic complications: Chinese visceral adiposity index and neck circumference. Cardiovasc Diabetol. 2020; 19(1):1-12. https://doi.org/10.1186/s12933-020-01095-4.

15. Zhou C, Zhan L, Yuan J, Tong X, Peng Y, Zha Y. Comparison of visceral, general and central obesity indices in the prediction of metabolic syndrome in maintenance hemodialysis patients. Eat Weight Disord. 2019:1-8. https:// doi.org/10.1007/s40519-019-00678-9.

16. Xia MF, Chen Y, Lin HD, Ma H, Li XM, Aleteng Q, et al. A indicator of visceral adipose dysfunction to evaluate metabolic health in adult Chinese. Sci Rep. 2016:6(1):38214. https://doi.org/10.1038/srep38214.

17. Ferreira FG, Juvanhol LL, da Silva DCG, Longo GZ. Visceral adiposity index is a better predictor of unhealthy metabolic phenotype than traditional adiposity measures: results from a population-based study. Public Health Nutr. 2019;22(9):1545-54. https://doi.org/10.1017/\$136898001800335X https://doi.org/10.1017/S136898001800335X

18. DeLong ER, DeLong DM, Clarke-Pearson DL. Comparing the areas under two or more correlated receiver operating characteristic curves: a nonparametric approach. Biometrics. 1988:44(3):837-45. https://doi.org/10.2307/2531595.

19. Stefan N, Schick F, Haring HU. Causes, characteristics, and consequences of metabolically unhealthy Normal weight in humans. Cell Metab. 2017;26(2): 292-300. https://doi.org/10.1016/j.cmet.2017.07.008.

20. Wu J, Gong L, Li Q, Hu J, Zhang S, Wang Y, et al. A novel visceral adiposity index for prediction of type 2 diabetes and pre-diabetes in Chinese adults: a 5-year prospective study. Sci Rep. 2017;7(1):13784. https://doi.org/10.1038/ s41598-017-14251-w.

21. Han M, Qie R, Li Q, Liu L, Huang S, Wu X, et al. Chinese visceral adiposity index, a novel indicator of visceral obesity for assessing the risk of incident hypertension in a prospective cohort study. Br J Nutr. 2020;126(4):1-9. https://doi.org/10.1017/S0007114520004298.

22. Xie X, Li Q, Zhang L, Ren W. Lipid accumulation product, visceral adiposity index, and Chinese visceral adiposity index as markers of Cardiometabolic risk in adult growth hormone deficiency patients: a cross-sectional study. Endocr Pract. 2018;24(1):33-9. https://doi.org/10.4158/EP-2017-0007.

23. Koloverou E, Panagiotakos DB, Kyrou I, Stefanadis C, Chrysohoou C, Georgousopoulou EN, et al. Visceral adiposity index outperforms common anthropometric indices in predicting 10-year diabetes risk: Results from the ATTICA study. Diabetes Metab Res Rev. 2019:e3161. https://doi.org/10.1002/ dmrr.3161.

24. Schousboe JT, Kats AM, Langsetmo L, Vo TN, Taylor BC, Schwartz AV, et al. Central obesity and visceral adipose tissue are not associated with incident atherosclerotic cardiovascular disease events in older men. J Am Heart Assoc. 2018;7(16):e009172. https://doi.org/10.1161/JAHA.118.009172.

25. Bawadi H, Abouwatfa M, Alsaeed S, Kerkadi A, Shi Z. Body Shape Index Is a Stronger Predictor of Diabetes. Nutrients. 2019;11(5). https://doi.org/10.3390/ nu11051018.

26. Geraci G, Zammuto M, Gaetani R, Mattina A, D'lgnoto F, Geraci C, et al. Relationship of a body shape index and body roundness index with carotid atherosclerosis in arterial hypertension. Nutr Metab Cardiovasc Dis. 2019; 29(8):822-9. https://doi.org/10.1016/j.numecd.2019.04.013.

27. Wang H, Liu A, Zhao T, Gong X, Pang T, Zhou Y, et al. Comparison of anthropometric indices for predicting the risk of metabolic syndrome and its components in Chinese adults: a prospective, longitudinal study. BMJ Open. 2017;7(9):e016062. https://doi.org/10.1136/bmjopen-2017-016062. 
28. Stefanescu A, Revilla L, Lopez T, Sanchez SE, Williams MA, Gelaye B. Using a body shape index (ABSI) and body roundness index (BRI) to predict risk of metabolic syndrome in Peruvian adults. J Int Med Res. 2020;48(1): 0300060519848854. https://doi.org/10.1177/0300060519848854.

29. Katsuki A, Sumida Y, Urakawa H, Gabazza EC, Murashima S, Maruyama N, et al. Increased visceral fat and serum levels of triglyceride are associated with insulin resistance in Japanese metabolically obese, normal weight subjects with normal glucose tolerance. Diabetes Care. 2003;26(8):2341-4. https://doi.org/10.2337/diacare.26.8.2341.

30. Yu X, Wang L, Zhang W, Ming J, Jia A, Xu S, et al. Fasting triglycerides and glucose index is more suitable for the identification of metabolically unhealthy individuals in the Chinese adult population: a nationwide study. J Diabetes Investig. 2019;10(4):1050-8. https://doi.org/10.1111/jdi.12975.

\section{Publisher's Note}

Springer Nature remains neutral with regard to jurisdictional claims in published maps and institutional affiliations.

Ready to submit your research? Choose BMC and benefit from:

- fast, convenient online submission

- thorough peer review by experienced researchers in your field

- rapid publication on acceptance

- support for research data, including large and complex data types

- gold Open Access which fosters wider collaboration and increased citations

- maximum visibility for your research: over $100 \mathrm{M}$ website views per year

At BMC, research is always in progress.

Learn more biomedcentral.com/submissions 